\title{
Monte Carlo model and single-scattering approximation of the propagation of polarized light in turbid media containing glucose
}

\author{
Xueding Wang, Gang Yao, and Lihong V. Wang
}

\begin{abstract}
We present a single-scattering model as well as a Monte Carlo model of the effect of glucose on polarized light in turbid media. Glucose alters the Mueller-matrix patterns of diffusely backscattered and forward-scattered light because glucose molecules rotate the polarization plane of linearly polarized light. For example, the angles of rotation in Mueller-matrix elements $S_{21}$ and $S_{12}$ are linearly related to the concentration of glucose and increase with the source-detector distance. In the nondiffusion regime, the two models agree well with each other. In the diffusion regime, the single-scattering model is invalid, but there still exists a linear relationship between the angles of rotation in the Mueller-matrix elements and the concentration of glucose, which is predicted by the Monte Carlo model. (C) 2002 Optical Society of America

OCIS codes: $\quad 290.0290,290.1350,290.4210,290.7050,260.5430$.
\end{abstract}

\section{Introduction}

Interest in research on the propagation of polarized light in randomly scattering media has increased recently because of the potential applications of polarized light in diverse fields. Many studies have suggested that relevant information can be obtained by analysis of the response of a medium to a polarized optical source. ${ }^{1-3}$ One can vary the polarization of the source and detect 16 independent intensity measurements, ${ }^{4,5}$ which are sufficient to yield the Mueller matrix that completely determines the transformation of any incident polarization state. Several research groups have developed numerical and analytical methods that describe the propagation of polarized light in scattering media. Ambirajan and Look ${ }^{6}$ developed a Monte Carlo model for multiple scattering of a polarized light beam. Hielscher et al.7,8 used a Stokes-Mueller approach to study multiply scattered polarized light. Bartel and Heilscher ${ }^{9}$ investigated the backward Mueller matrix

The authors are with the Optical Imaging Laboratory, Biomedical Engineering Program, Texas A\&M University, 3120 TAMU, College Station, Texas 77843-3120. L. Wang's e-mail address is LWang@tamu.edu.

Received 15 March 2001; revised manuscript received 14 September 2001.

0003-6935/02/040792-10\$15.00/0

(C) 2002 Optical Society of America of highly scattering media. Yao and Wang10 produced the animation sequences of propagation of polarized light in turbid media. The symmetry of the polarization patterns from incoherent backscattered light as well as the relationship among the 16 matrix elements have also been studied in detail and can be used to predict the patterns and to expedite simulations. ${ }^{3,11,12}$ All these groups of researchers used Monte Carlo algorithms based on the Stokes-Mueller formulation.

Optical approaches to studying turbid media in the presence of chiral components have generated interest because of their potential use in noninvasive glucose monitoring for diabetes patients. Tissue glucose levels have been correlated with scattering coefficients based on changes in the refractive indices of extracellular fluids. ${ }^{13-15}$ Mehrubeoglu et al. measured the cross-polarization patterns with glucose in turbid media. ${ }^{16}$ Zhou and Schmitt, ${ }^{17}$ Cote and coworkers, ${ }^{18,19}$ Chou et al., ${ }^{20,21}$ and $\mathrm{Pu}$ et al. ${ }^{22}$ used highly sensitive polarimetric technology to discern the small rotations in polarized light associated with physiological glucose levels. All their methodologies are designed to measure glucose concentrations in the aqueous humor by taking advantage of the low scattering coefficient of the eye. However, there is a time lag between the glucose concentrations in the blood and in the aqueous humor during the rapid shifting of blood glucose concentration, and it is difficult to couple light through the eye. Ablitt et al. 
examined the behavior of polarized light scattered by turbid media that contained small chiral spheroidal particles, using Monte Carlo simulations. ${ }^{23}$ Studinski and Vitkin studied the correlation between concentrations of chiral molecules in turbid media and the properties of polarized light emerging from turbid media, such as the optical rotation of the linearly polarized fractions and the degrees of polarization. ${ }^{24}$

As a chiral material, glucose has preferential handedness because it has an asymmetric molecular structure. As a result, glucose interacts differently with right and left circularly polarized light, resulting in rotation of the linear polarization plane. ${ }^{25}$ In a highly scattering condition, when the direction and the polarization of the incident beam are randomized, it is difficult to detect the rotation of the linear polarization plane because the fraction of informationcontaining light is small compared with the amount of randomized information-degrading light. With a poor signal/noise ratio, detection of the small rotations of the polarization plane caused by glucose in physiological levels is quite difficult.

We found that the change in the polarization of reemitted light caused by glucose can also be observed from Mueller-matrix patterns of the turbid media. The Mueller-matrix patterns of diffusely backscattered and forward-scattered light are altered in relation to the concentration of glucose levels in turbid media, which makes it possible to monitor the concentration of glucose noninvasively in highly scattering tissues other than the low-scattering aqueous humor of the eye.

In this paper we present a theoretical study of the contribution of glucose to Mueller-matrix patterns of turbid media. In the following analysis it is assumed that the scattering events are independent, and no coherence effects are considered. First, we introduce our single-scattering approximation. We apply this model to predict the Mueller-matrix patterns of turbid media that contain glucose; we use formulas from single-scattering approximations to calculate the rotations of some Mueller-matrix elements $S_{i, j}(i=1,2,3,4 ; j=1,2,3,4)$. Second, we implement Monte Carlo simulations based on Mie theory. The two models are compared in the singlescattering regime. The correlation between the rotation angles of the matrix elements and the concentration of glucose are discussed.

\section{Single-Scattering Approximation}

In this section we assume that the scattering of light is incoherent and that reemitted light comes primarily from single-scattering events in the medium, which means that light exits the medium after one scattering event. Any interaction between light and the medium's surface is neglected. We also assume that every photon packet that exits the medium reaches the detector, no matter its propagation angle and position as it exits the medium.

Our single-scattering model for propagation of polarized light is similar to the numerical method introduced by Rakovic et $a l^{3}$ The geometry of a

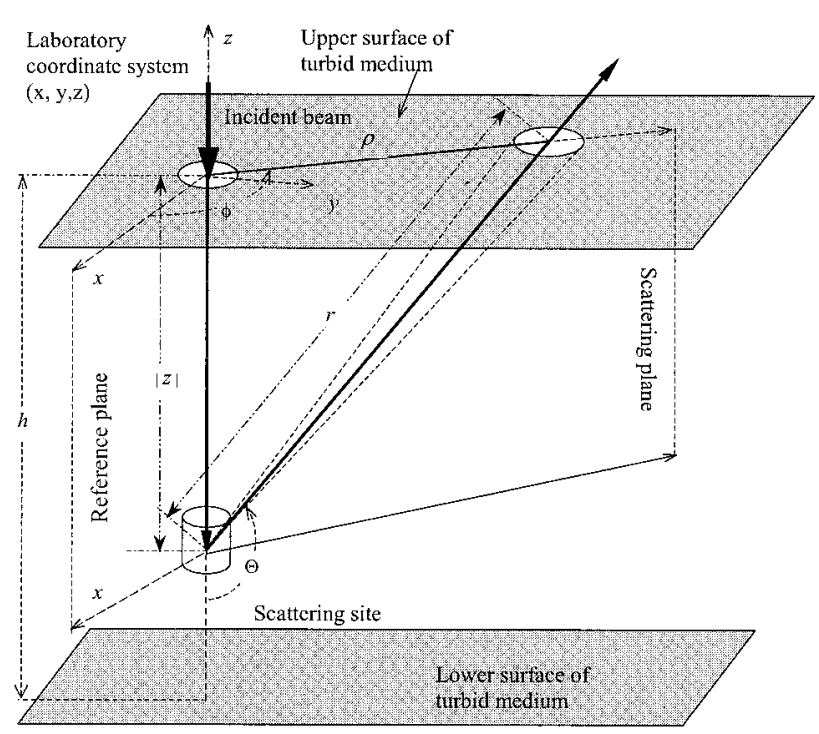

Fig. 1. Geometry of a single-scattering event.

backward single-scattering event is shown in Fig. 1 . A narrow pencillike beam propagates downward along the $z$ axis into a plane-parallel medium with thickness $h$. Scattering events occur at the lower half-space of the medium, $-h \leq z \leq 0$. Inasmuch as we assume that the light is scattered in the turbid medium by spheres, Mie theory can be used to describe the scattering process, and a scattering matrix takes the form ${ }^{26}$

$$
\mathbf{M}(\Theta)=\left[\begin{array}{cccc}
a(\Theta) & b(\Theta) & 0 & 0 \\
b(\Theta) & a(\Theta) & 0 & 0 \\
0 & 0 & d(\Theta) & -e(\Theta) \\
0 & 0 & e(\Theta) & d(\Theta)
\end{array}\right]
$$

When the spheres are small compared with the wavelength of the light (as in Rayleigh and dipole scattering), the above coefficients can be reduced to ${ }^{12}$

$$
\begin{aligned}
& a(\Theta)=\frac{3}{16 \pi}\left(1+\cos ^{2} \Theta\right), \\
& b(\Theta)=\frac{3}{16 \pi}\left(-1+\cos ^{2} \Theta\right), \\
& d(\Theta)=\frac{3}{8 \pi} \cos \Theta, \quad e(\Theta)=0 .
\end{aligned}
$$

A rotation matrix, $\mathbf{R}_{1}(\phi)$, transforms the Stokes vector in the reference plane into one in the scattering plane before scattering. Another rotation matrix, $\mathbf{R}_{2}(\phi)$, transforms the Stokes vector of light back into the reference plane from the scattering plane when light exits the sample surface. Both of these rotation matrices have the following form:

$$
\mathbf{R}(\phi)=\left[\begin{array}{cccc}
1 & 0 & 0 & 0 \\
0 & \cos (2 \phi) & -\sin (2 \phi) & 0 \\
0 & \sin (2 \phi) & \cos (2 \phi) & 0 \\
0 & 0 & 0 & 1
\end{array}\right]
$$


The Mueller matrix that represents the contribution of glucose to a certain propagation path can be considered the matrix for circular birefringence $\mathbf{R}(\theta)$ :

$$
\mathbf{R}(\theta)=\left[\begin{array}{cccc}
1 & 0 & 0 & 0 \\
0 & \cos (2 \theta) & -\sin (2 \theta) & 0 \\
0 & \sin (2 \theta) & \cos (2 \theta) & 0 \\
0 & 0 & 0 & 1
\end{array}\right]
$$

elements of the forward-scattered and backscattered Mueller matrix. For backscattering,

$$
\begin{aligned}
\mathbf{S}(\rho, \phi)= & \mu_{s} \int_{-h}^{0} \mathrm{~d} z\left\{\left(1 / r^{2}\right) \exp \left[-\mu_{T}(|z|+r)\right]\right. \\
& \left.\times \mathbf{R}_{2}(\phi) \mathbf{T}\left(\theta,{ }_{1}, \theta_{2}, \Theta\right) \mathbf{R}_{1}(\phi)\right\}
\end{aligned}
$$

$T_{i j}\left(\theta_{1}, \theta_{2}, \Theta\right)$
$\quad=\left[\begin{array}{cccc}a & b \cos 2 \theta_{1} & -b \sin 2 \theta_{1} & 0 \\ b \cos 2 \theta_{2} & a \cos 2 \theta_{2} \cos 2 \theta_{1}-d \sin 2 \theta_{2} \sin 2 \theta_{1} & -d \sin 2 \theta_{2} \sin 2 \theta_{1}-a \sin 2 \theta_{2} \sin 2 \theta_{1} & e \sin 2 \theta_{2} \\ b \sin 2 \theta_{2} & a \sin 2 \theta_{2} \cos 2 \theta_{1}+d \cos 2 \theta_{2} \sin 2 \theta_{1} & -a \sin 2 \theta_{2} \sin 2 \theta_{1}+d \cos 2 \theta_{2} \cos 2 \theta_{1} & -e \cos 2 \theta_{2} \\ 0 & e \sin 2 \theta_{1} & e \cos 2 \theta_{1} & d\end{array}\right]$,

where $\theta$ denotes the optical rotation caused by glucose molecules, which is the product of the optical rotation degree $[(\mathrm{ORD})$ the specific rotation of glucose for a certain wavelength of light], the path length of light, and the concentration of glucose $\alpha$.

If $\mathbf{P}_{0}$ is the Stokes vector of the incident laser beam with respect to the laboratory coordinate system $(x, y$, $z$ ), backscattered Stokes vector $\mathbf{I}^{\text {bs }}$ of the total radiance received by the detector at the position $(\rho, \phi, z=$ 0 ) on the upper surface of medium will be

$$
\begin{aligned}
\mathbf{I}^{\mathrm{bs}}(\rho, \phi)= & \mu_{s} \int_{-h}^{0} \mathrm{~d} z\left\{\left(1 / r^{2}\right) \exp \left[-\mu_{T}(|z|+r)\right]\right. \\
& \left.\times \mathbf{R}_{2}(\phi) \mathbf{R}\left(\theta_{2}\right) \mathbf{M}(\Theta) \mathbf{R}_{1}\left(\theta_{1}\right) \mathbf{R}(\phi) \mathbf{P}_{0}\right\},
\end{aligned}
$$

where $r=\sqrt{\rho^{2}+z^{2}} ; \tan \Theta=\rho / z ; \mu_{s}$ and $\mu_{T}$ are the scattering coefficient and the extinction coefficient, respectively; and $\theta_{1}=z \times \alpha \times \mathrm{ORD}$ and $\theta_{2}=r \times \alpha \times$ ORD are the angles of rotation according to paths $z$ and $r$, respectively.

Similarly, forward-scattered Stokes vector $\mathbf{I}^{\mathrm{fs}}$ of the total radiance received by the detector at position ( $\rho$, $\phi, z=h$ ) on the bottom surface of the medium will be

$$
\begin{aligned}
\mathbf{I}^{\mathrm{fs}}(\rho, \phi)= & \mu_{s} \int_{-h}^{0} \mathrm{~d} z\left\{\left(1 / r^{2}\right) \exp \left[-\mu_{T}(|z|+r)\right]\right. \\
& \left.\times \mathbf{R}_{2}(-\phi) \mathbf{R}\left(\theta_{2}\right) \mathbf{M}(\Theta) \mathbf{R}\left(\theta_{1}\right) \mathbf{R}_{1}(\phi) \mathbf{P}_{0}\right\}
\end{aligned}
$$

The only difference from the backscattered Stokes vector that should be noticed is the appearance of the negative sign in $\mathbf{R}_{2}(-\phi)$ because the second rotation that transforms the Stokes vector back to the reference plane should be anticlockwise, which is a negative rotation compared with the first positive rotation in $\mathbf{R}_{1}(\phi)$.

From Eqs. (1)-(6) we can obtain the formula for all where $a, b, d$, and $e$ are $a(\Theta), b(\Theta), d(\Theta)$, and $e(\Theta)$, respectively, and subscripts $i$ and $j$ are indexed from 1 to 4 .

From Eqs. (7) and (8) we can predict the pattern of every element in the effective backscattering Mueller matrix of a turbid medium with a certain concentration of glucose. As for the forward-scattering Mueller-matrix elements, the deduction process is the same.

For a glucose concentration $\alpha=0$ we can simplify the Mueller-matrix expression obtained by the single-scattering approximation as follows:

$$
\begin{aligned}
S_{i j}(\rho, \phi)= & \mu_{s} \int_{-h}^{0} \frac{\mathrm{d} z}{r^{2}} \exp \left[-\mu_{T}(|z|+r)\right] F_{i j}, \\
F_{11}= & a(\Theta), \\
F_{12}= & b(\Theta) \cos (2 \phi), \\
F_{13}= & -b(\Theta) \sin (2 \phi), \\
F_{14}= & 0, \\
F_{21}= & F_{12}, \\
F_{22}= & a(\Theta) \cos (2 \phi) \cos (2 \phi)-d(\Theta) \sin (2 \phi) \\
& \times \sin (2 \phi) \\
= & \frac{a(\Theta)-d(\Theta)}{2} \\
& +\frac{a(\Theta)+d(\Theta)}{2} \cos (4 \phi), \\
F_{23}= & -a(\Theta) \cos (2 \phi) \sin (2 \phi) \\
& -d(\Theta) \cos (2 \phi) \sin (2 \phi) \\
= & -[a(\Theta)+d(\Theta)] \sin (4 \phi), \\
F_{24}= & e(\Theta) \sin (2 \phi), \\
F_{31}= & -F_{13},
\end{aligned}
$$




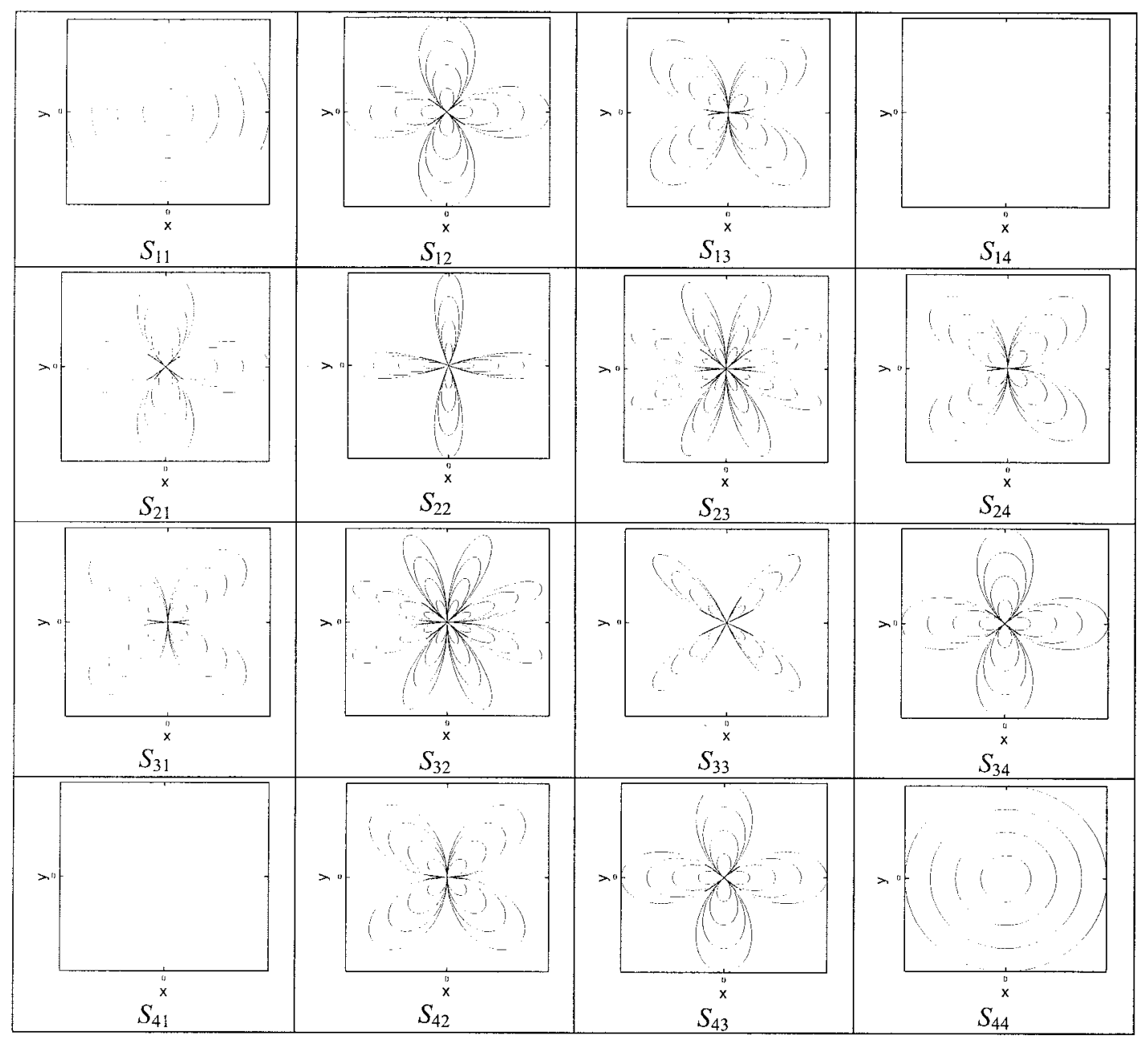

Fig. 2. Single-scattering profiles of the backscattering Mueller-matrix patterns from a turbid medium.

$$
\begin{aligned}
F_{32}= & -F_{23}, \\
F_{33}= & -a(\Theta) \sin (2 \phi) \sin (2 \phi) \\
& +d(\Theta) \cos (2 \phi) \cos (2 \phi) \\
= & \frac{-a(\Theta)+d(\Theta)}{2} \\
& +\frac{a(\Theta)+d(\Theta)}{2} \cos (4 \phi), \\
F_{34}= & -e(\Theta) \cos (2 \phi), \\
F_{41}= & 0, \\
F_{42}= & F_{24}, \\
F_{43}= & -F_{34}, \\
F_{44}= & d(\Theta) .
\end{aligned}
$$

Based on Eqs. (9), Fig. 2 shows profiles of the azimuthal dependence of the backscattering Muellermatrix patterns from turbid media without glucose. The results in Fig. 2 are similar to the analytical expressions given by Rakovic et al..$^{3}$ and Rakovic and Kattawar. ${ }^{12}$ The single-scattering approximation model, at least qualitatively, provides us an effective method with which to emulate the scattering patterns from a suspension of polystyrene spheres as measured in experiments. ${ }^{3,11}$

Among the 16 elements of the backscattering Mueller matrix, we discuss $S_{12}, S_{13}, S_{21}$, and $S_{31}$ in detail. We expand them into the following equations:

$$
\begin{aligned}
S_{12}(\rho, \phi)= & \mu_{s} \cos (2 \phi) \int_{-h}^{0} \mathrm{~d} z\left\{\left(1 / r^{2}\right)\right. \\
& \left.\times \exp \left[-\mu_{T}(|z|+r)\right] \cos \left(2 \theta_{1}\right) b(\Theta)\right\} \\
& -\mu_{s} \sin (2 \phi) \int_{-h}^{0} \mathrm{~d} z\left\{\left(1 / r^{2}\right)\right. \\
& \left.\times \exp \left[-\mu_{T}(|z|+r)\right] \sin \left(2 \theta_{1}\right) b(\Theta)\right\}, \\
S_{13}(\rho, \phi)= & \mu_{s} \sin (2 \phi) \int_{-h}^{0} \mathrm{~d} z\left\{\left(1 / r^{2}\right)\right. \\
& \left.\times \exp \left[-\mu_{T}(|z|+r)\right] \cos \left(2 \theta_{1}\right) b(\Theta)\right\} \\
& +\mu_{s} \cos (2 \phi) \int_{-h}^{0} \mathrm{~d} z\left\{\left(1 / r^{2}\right)\right.
\end{aligned}
$$




$$
\begin{aligned}
& \left.\times \exp \left[-\mu_{T}(|z|+r)\right] \sin \left(2 \theta_{1}\right) b(\Theta)\right\}, \\
S_{21}(\rho, \phi)= & \mu_{s} \cos (2 \phi) \int_{-h}^{0} \mathrm{~d} z\left\{\left(1 / r^{2}\right)\right. \\
& \left.\times \exp \left[-\mu_{T}(|z|+r)\right] \cos \left(2 \theta_{2}\right) b(\Theta)\right\} \\
& -\mu_{s} \sin (2 \phi) \int_{-h}^{0} \mathrm{~d} z\left\{\left(1 / r^{2}\right)\right. \\
& \left.\times \exp \left[-\mu_{T}(|z|+r)\right] \sin \left(2 \theta_{2}\right) b(\Theta)\right\}, \\
S_{31}(\rho, \phi)= & \mu_{s} \sin (2 \phi) \int_{-h}^{0} \mathrm{~d} z\left\{\left(1 / r^{2}\right)\right. \\
& \left.\times \exp \left[-\mu_{T}(|z|+r)\right] \cos \left(2 \theta_{2}\right) b(\Theta)\right\} \\
& +\mu_{s} \cos (2 \phi) \int_{-h}^{0} \mathrm{~d} z\left\{\left(1 / r^{2}\right)\right. \\
& \left.\times \exp \left[-\mu_{T}(|z|+r)\right] \sin \left(2 \theta_{2}\right) b(\Theta)\right\} .
\end{aligned}
$$

There are two integrals from $-h$ to 0 in each of Eqs. (10) that can be reduced to a sine and a cosine function. The following simple expressions will be obtained:

$$
\begin{aligned}
& S_{12}(\rho, \phi)=K_{1}\left(\rho, \alpha, \mu_{T}, \mu_{s}\right) \cos \left(2 \phi+2 \bar{\theta}_{1}\right), \\
& S_{13}(\rho, \phi)=K_{1}\left(\rho, \alpha, \mu_{T}, \mu_{s}\right) \sin \left(2 \phi+2 \bar{\theta}_{1}\right), \\
& S_{21}(\rho, \phi)=K_{2}\left(\rho, \alpha, \mu_{T}, \mu_{s}\right) \cos \left(2 \phi+2 \bar{\theta}_{2}\right), \\
& S_{31}(\rho, \phi)=K_{2}\left(\rho, \alpha, \mu_{T}, \mu_{s}\right) \sin \left(2 \phi+2 \bar{\theta}_{2}\right),
\end{aligned}
$$

where

$$
\begin{aligned}
K_{1}\left(\rho, \phi, \alpha, \mu_{T}, \mu_{s}\right)= & \left(\left\{\int_{-h}^{0} \frac{\mathrm{d} z}{r^{2}} \exp \left[-\mu_{T}(|z|+r)\right]\right.\right. \\
& \left.\times \sin \left(2 \theta_{1}\right) b(\Theta)\right\}^{2} \\
& +\left\{\int_{-h}^{0} \frac{\mathrm{d} z}{r^{2}} \exp \left[-\mu_{T}(|z|+r)\right]\right. \\
& \left.\left.\times \cos \left(2 \theta_{1}\right) b(\Theta)\right\}^{2}\right)^{1 / 2}, \\
K_{2}\left(\rho, \phi, \alpha, \mu_{T}, \mu_{s}\right)= & \left\{\left\{\int_{-h}^{0} \frac{\mathrm{d} z}{r^{2}} \exp \left[-\mu_{T}(|z|+r)\right]\right.\right. \\
& \left.\times \sin \left(2 \theta_{2}\right) b(\Theta)\right\}^{2} \\
& +\left\{\int_{-h}^{0} \frac{\mathrm{d} z}{r^{2}} \exp \left[-\mu_{T}(|z|+r)\right]\right. \\
& \left.\left.\times \cos \left(2 \theta_{2}\right) b(\Theta)\right\}^{2}\right)^{1 / 2},
\end{aligned}
$$

$\tan \left(2 \bar{\theta}_{1}\right)$

$$
=\frac{\int_{-h}^{0} \mathrm{~d} z\left\{\left(1 / r^{2}\right) \exp \left[-\mu_{T}(|z|+r)\right] \sin \left(2 \theta_{1}\right) b(\Theta)\right\}}{\int_{-h}^{0} \mathrm{~d} z\left\{\left(1 / r^{2}\right) \exp \left[-\mu_{T}(|z|+r)\right] \cos \left(2 \theta_{1}\right) b(\Theta)\right\}},
$$

$\tan \left(2 \bar{\theta}_{2}\right)$

$$
=\frac{\int_{-h}^{0} \mathrm{~d} z\left\{\left(1 / r^{2}\right) \exp \left[-\mu_{T}(|z|+r)\right] \sin \left(2 \theta_{2}\right) b(\Theta)\right\}}{\int_{-h}^{0} \mathrm{~d} z\left\{\left(1 / r^{2}\right) \exp \left[-\mu_{T}(|z|+r)\right] \cos \left(2 \theta_{2}\right) b(\Theta)\right\}} .
$$

When the concentration of glucose equals zero, these four elements will be

$$
\begin{aligned}
& S_{12}(\rho, \phi)=K_{1}\left(\rho, \alpha, \mu_{T}, \mu_{s}\right) \cos (2 \phi), \\
& S_{13}(\rho, \phi)=K_{1}\left(\rho, \alpha, \mu_{T}, \mu_{s}\right) \sin (2 \phi), \\
& S_{21}(\rho, \phi)=K_{2}\left(\rho, \alpha, \mu_{T}, \mu_{s}\right) \cos (2 \phi), \\
& S_{31}(\rho, \phi)=K_{2}\left(\rho, \alpha, \mu_{T}, \mu_{s}\right) \sin (2 \phi),
\end{aligned}
$$

which are cloverleaflike patterns shown in Fig. 2.

From Eqs. (11)-(14) one can see that the glucose in turbid media causes the phase shifts $\bar{\theta}_{1}$ and $\bar{\theta}_{2}$ in the azimuthal functions of matrix elements $S_{12}, S_{13}$ and $S_{21}, S_{31}$, respectively. Phase shifts $\bar{\theta}_{1}$ and $\bar{\theta}_{2}$ in cosine and sine functions in Eqs. (11) are expressed as rotations of the matrix patterns, which are shown in Fig. 3. The angles of rotation, $\bar{\theta}_{1}$, of elements $S_{12}$ and $S_{13}$ and $\bar{\theta}_{2}$, of elements $S_{21}$ and $S_{31}$, are determined mainly by the integrations of $\theta_{1}$, which depend only on the path along $z$, and $\theta_{2}$, which depend only on the path along $r$, where both $\theta_{1}$ and $\theta_{2}$ are proportional to the concentration of glucose. One can also expect that, when source-detector distance $\rho$ increases, $\bar{\theta}_{1}$ and $\bar{\theta}_{2}$ will increase at different rates. During detection of positions far away from the incident point $\left(\rho>1 / \mu_{T}\right), \bar{\theta}_{1}$ approaches a constant while $\bar{\theta}_{2}$ has a nearly linear relationship to source-detector distance $\rho$. When $\rho$ approaches $0, b(\Theta)$ in Eqs. (11)(14) approaches 0; consequently, $S_{12}, S_{13}, S_{21}$, and $S_{31}$ approach 0 and indicate no rotations.

\section{Monte Carlo Simulation}

We used a Monte Carlo simulation based on Mie theory to simulate the effective back-scattering Mueller matrix of a scattering medium containing spherical scatters. Our Monte Carlo code is based on an algorithm developed previously. ${ }^{10,27}$ The scattering Mueller matrix, $\mathbf{M}(\Theta)$, is given by Mie theory instead of by the Rayleigh scattering described in Section 2, whereas $\mathbf{R}(\phi)$ and $\mathbf{R}(\theta)$ remain the same. ${ }^{26}$ Element 

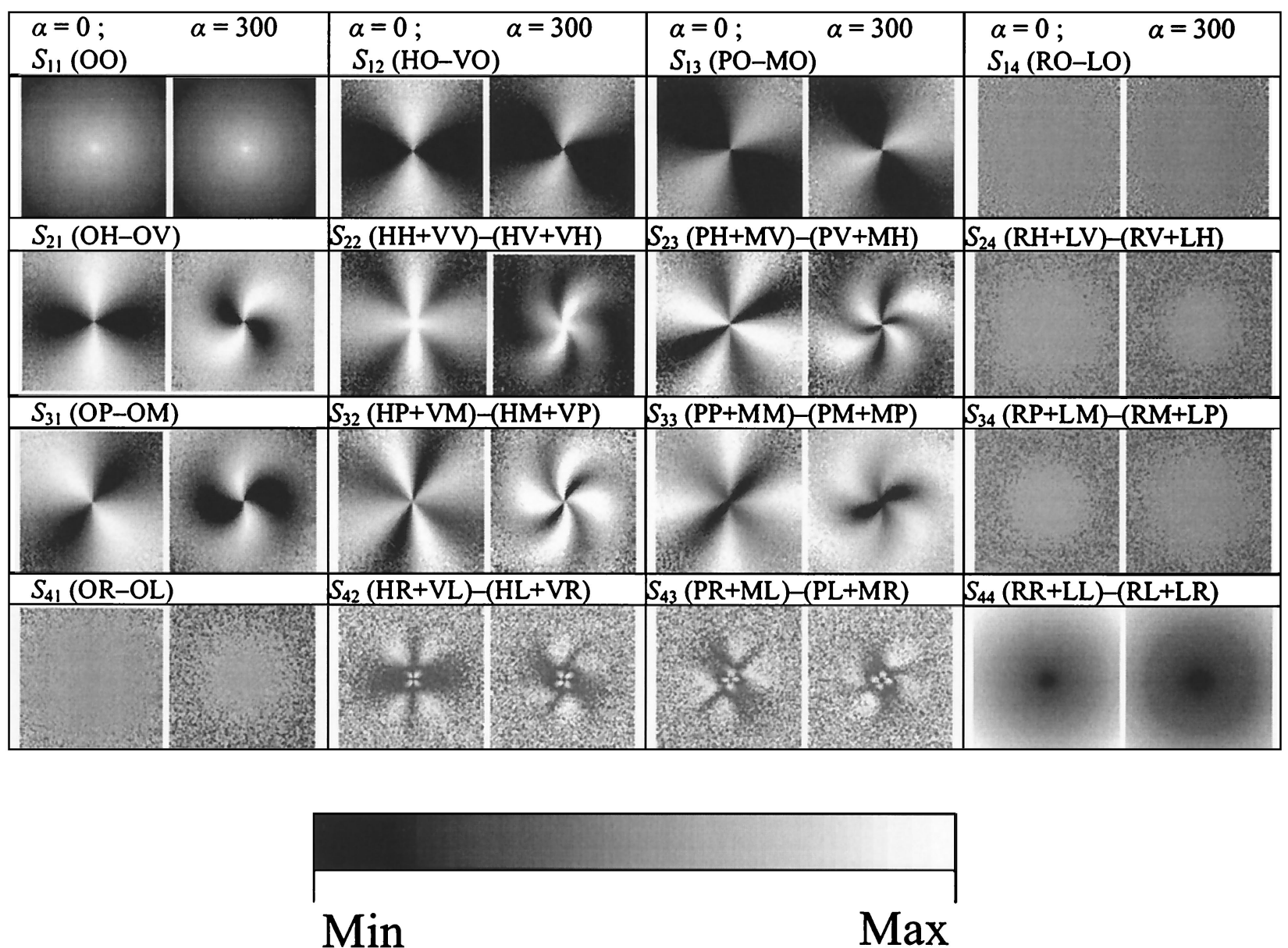

Fig. 3. Comparison of results of a Monte Carlo model of a backscattering Mueller matrix from turbid media with and without glucose. The size of each intensity map is $4 \mathrm{~cm} \times 4 \mathrm{~cm}$. Absorption coefficient $\mu_{a}, 0.01 \mathrm{~cm}^{-1}$; scattering coefficient $\mu_{s}, 1 \mathrm{~cm}^{-1}$; concentration of glucose $\alpha, 300 \mathrm{~g} / \mathrm{dL}$. The symbols to describe $S_{i j}$ consist of double-polarization states with the input polarization state denoted by the left-hand letter and the output polarization state denoted by the right-hand letter. $\mathrm{O}$, unpolarized light; $\mathrm{H}$, horizontally linear polarization; $\mathrm{V}$, vertically linear polarization; $\mathrm{P}$, linear polarization oriented in the $+45^{\circ}$ direction; $\mathrm{M}$, linear polarization oriented in the $-45^{\circ}$ direction; R, right-circular polarization; L, left-circular polarization.

$a(\Theta)$ in $\mathbf{M}(\Theta)$ satisfies the following normalization requirement:

$$
2 \pi \int_{0}^{\pi} a(\Theta) \sin (\Theta) \mathrm{d} \Theta=1
$$

For each scattering event, the sampling of polar angle $\Theta$ and azimuthal angle $\phi$ satisfies the following probability-density function:

$\rho(\Theta, \phi)=a(\Theta)+b(\Theta)\left[S_{2} \cos (2 \phi)+S_{3} \sin (2 \phi)\right] / S_{1}$,

which is a function of incident Stokes vector $\mathbf{S}=\left[S_{1}\right.$, $\left.S_{2}, S_{3}, S_{4}\right]^{T}$.

For statistical results, large quantities of photon packets $\left(10^{7}\right)$ are traced from a pencillike beam along the $z$ axis. In the following simulations, the radius of spheres in the medium is $125 \mathrm{~nm}$, the refractive index $n_{0}$ of the medium is 1.33 , the refractive index $n_{\mathrm{s}}$ of polystyrene spherical particles is 1.57 , the wavelength of light $\lambda_{0}$ in vacuo is $594 \mathrm{~nm}$, and the specific rotation of glucose at this wavelength is $\mathrm{ORD}=52.7^{\circ}$ $\mathrm{dm}^{-1}(\mathrm{~g} / \mathrm{mL})^{-1} .28$

The Monte Carlo simulation results for the backscattering matrix are shown in Fig. 3. Sixteen matrix elements of the turbid medium without glucose are compared with the matrix elements of the turbid medium with glucose while other optical parameters are kept the same. The size of each intensity map in Fig. 3 is $4 \mathrm{~cm} \times 4 \mathrm{~cm}$. To ensure that most photon packets will exit the medium from the upper or the bottom surface after one scattering event, absorption coefficient $\mu_{a}$ is set at $0.01 \mathrm{~cm}^{-1}$ and scattering coefficient $\mu_{s}$ is set at $1 \mathrm{~cm}^{-1}$. The thickness of the turbid medium is set at $1 \mathrm{~cm}$. Through calculation, anisotropic factor $g$ is 0.134 ; the mean free path is $0.99 \mathrm{~cm}$, and the transport mean free path is 1.142 $\mathrm{cm}$. Because the rotations in polarized light caused by the physiological glucose levels are small, we set glucose concentration $\alpha$ at $300(\mathrm{~g} / \mathrm{dL})$ to permit the differences in the matrix patterns to be observed.

The right-hand map in each cell in Fig. 3 shows that some intensity patterns of the matrix elements 


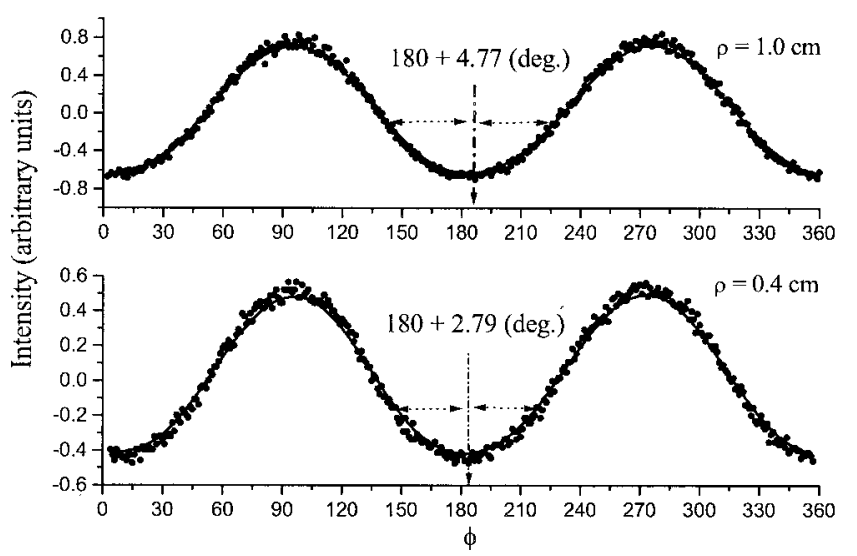

(a)

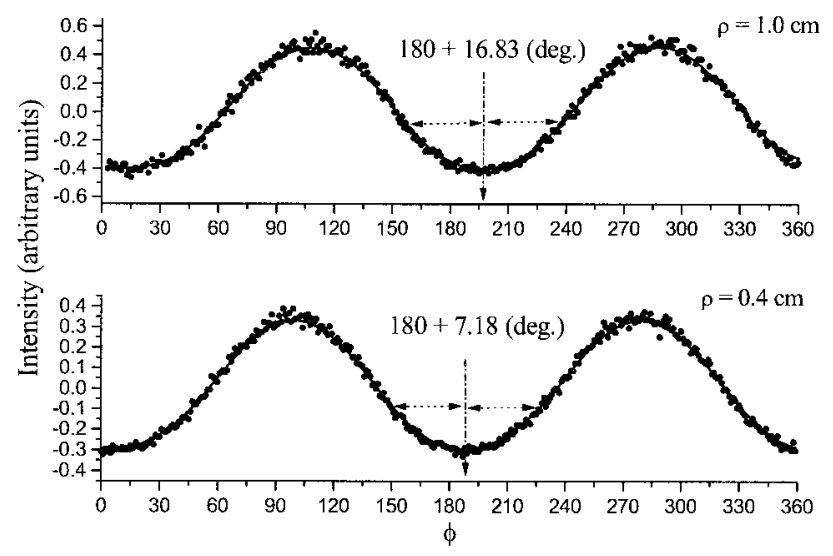

(b)

Fig. 4. Results of single scattering (solid curves) and Monte Carlo (scattered symbols) models of the azimuthal dependence of backward Mueller-matrix pattern elements (a) $S_{12}$ and (b) $S_{21}$. The source-detector distances are $0.4 \mathrm{~cm}$ and $1.0 \mathrm{~cm}$. Absorption coefficient $\mu_{a}, 0.01 \mathrm{~cm}^{-1}$; scattering coefficient $\mu_{s}, 1 \mathrm{~cm}^{-1}$; concentration of glucose, $300 \mathrm{~g} / \mathrm{dL}$.

have obvious rotations about the incident point, which is at the center of each map. Figure 4 shows a comparison of the azimuthal dependence of $S_{12}$ and $S_{21}$ along 0.4- and 1-cm source-detector distances. Intensity distributions of $S_{12}$ and $S_{21}$ fit well with the cosine functions of two times azimuth angle $\phi$ (from 0 to $360^{\circ}$ ). The rotation of the patterns caused by glucose can be seen from the phase shifts of the cosine functions, which are marked in Fig. 4 and explained in detail in Eqs. (11) as $\bar{\theta}_{1}$ and $\bar{\theta}_{2}$, respectively. One may notice that the rotation of $S_{21}$ and $S_{31}$ increases more rapidly than the rotation of $S_{12}$ and $S_{13}$ as source-detector distance $\rho$ increases, in good agreement with our discussion above of the single-scattering approximation. In Section 4 we compare the numerical results from the Monte Carlo simulation and the singlescattering model.

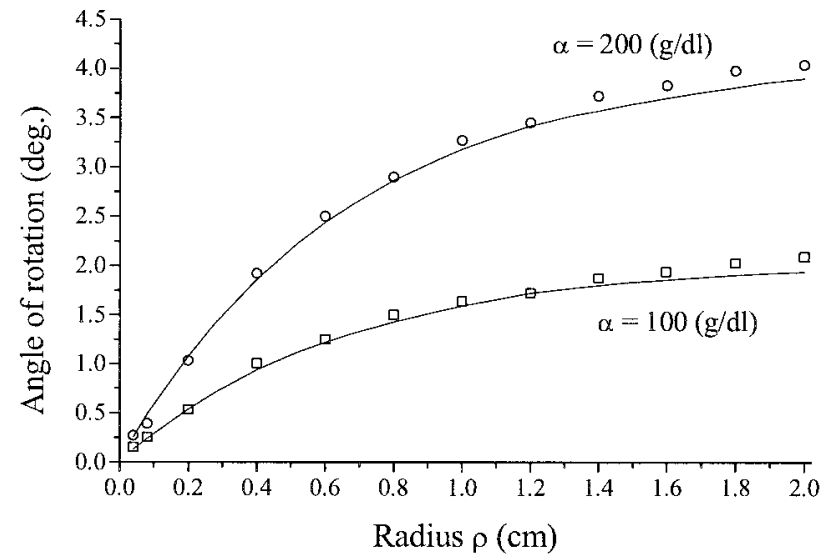

(a)

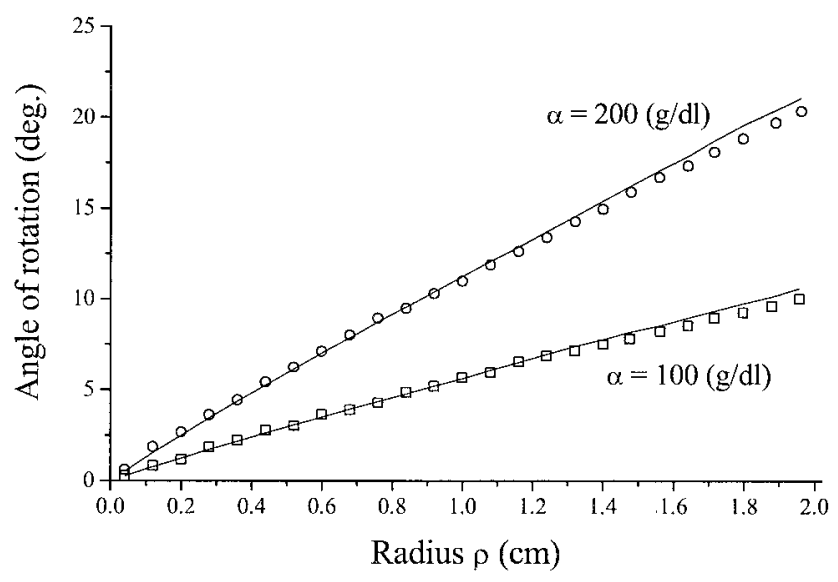

(b)

Fig. 5. Rotation angles $\bar{\theta}_{1}$ and $\bar{\theta}_{2}$ for changes in backward Mueller-matrix elements $S_{12}$ (a) and (b) $S_{21}$ with changes in the source-detector distance. Solid curves, results from the singlescattering model; symbols, results from the Monte Carlo model. $\mu_{s}, 10 \mathrm{~cm}^{-1} ; \mu_{a}, 0.1 \mathrm{~cm}^{-1}$; thickness of the sample $h, 1 \mathrm{~cm} ; \alpha$, concentrations of glucose.

\section{Comparison and Discussion}

Through a Mie-theory-based Monte Carlo simulation, we obtain intensity maps for the 16 matrix elements in polar coordinates. For each source-detector distance, the intensities of the $S_{12}$ and $S_{21}$ elements about a circle are discrete quantities with respect to the azimuth angle from $0^{\circ}$ to $360^{\circ}$. The azimuthaldependent distributions of intensities of $S_{12}$ and $S_{21}$ fit the cosine functions with phase shifts $\bar{\theta}_{1}$ and $\bar{\theta}_{2}$, respectively, which can be compared with the results calculated from Eqs. (12) based on the singlescattering approximation.

In the simulations shown in Figs. 5-8, the optical parameters of the turbid media are as follows: $\mu_{s}$ is $10 \mathrm{~cm}^{-1}, \mu_{a}$ is $0.1 \mathrm{~cm}^{-1}$, anisotropic factor $g$ is calculated to be 0.134 , and the thickness of the sample, $h$, is $1 \mathrm{~cm}$.

Figures 5(a) and 5(b) show the rotation of elements $S_{12}$ and $S_{21}$, respectively, as they change with 




Fig. 6. Distribution of backscattered light intensities on scattering events of light $\left(\mu_{a}, 0.1 \mathrm{~cm}^{-1} ; \mu_{s}, 10 \mathrm{~cm}^{-1}\right.$; thickness of the turbid medium, $1 \mathrm{~cm}$ ).

source-detector distance $\rho$ from 0 to $2 \mathrm{~cm}$. Glucose concentrations are set at 200 and $100(\mathrm{~g} / \mathrm{dL})$. The degrees of rotation obtained by the Monte Carlo sim-



(a)



(b)

Fig. 7. Monte Carlo simulated rotation of (a) matrix element $S_{12}$ $\bar{\theta}_{1}$ and (b) matrix element $S_{21}, \bar{\theta}_{2} . \quad \mu_{s}, 10 \mathrm{~cm}^{-1} ; \mu_{a}, 0.1 \mathrm{~cm}^{-1}$; thickness of the sample $h, 1 \mathrm{~cm}$.

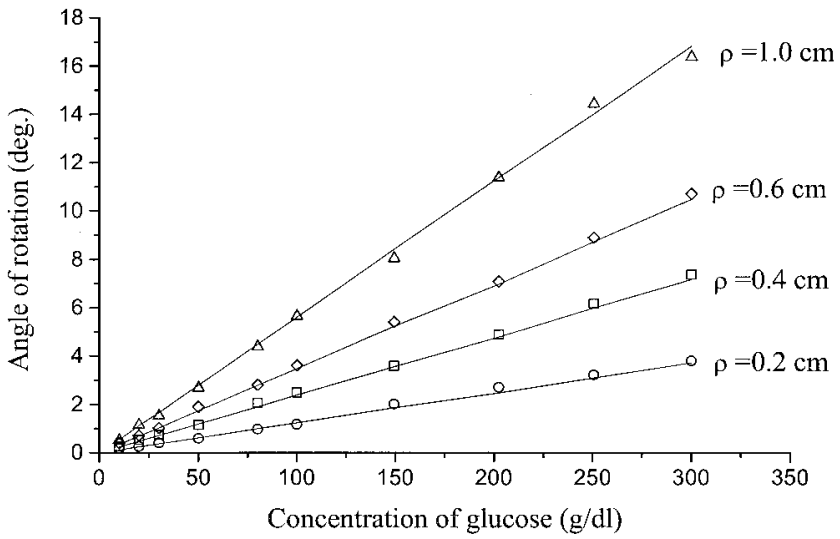

Fig. 8. Rotation angles $\bar{\theta}_{2}$ of changes in backward Mueller-matrix element $S_{21}$ with changes in the concentration of glucose. Solid curves, results from the single-scattering model; symbols, results from the Monte Carlo model. Source-detector distances $\rho$ are shown. $\mu_{s}, 10 \mathrm{~cm}^{-1} ; \mu_{a}, 0.1 \mathrm{~cm}^{-1}$; thickness of the sample $h, 1 \mathrm{~cm}$.

ulation (symbols) are in good agreement with the predicted results from the single-scattering model (solid curves) when source-detector distance $\rho$ is $0-1.3 \mathrm{~cm}$. In this period, $\bar{\theta}_{2}$, the rotation of element $S_{21}$, increases with source-detector distance $\rho$ in a nearly linear relationship, whereas $\bar{\theta}_{1}$, the rotation of $S_{12}$, increases at a comparatively lower rate. According to Fig. 1, the path lengths of light increase with the source-detector distance. When the detection position is far away from the incident point, the contribution from multiply scattered photons to the total backscattered light cannot be ignored. In Fig. 5 a discrepancy appears between the results of the Monte Carlo simulation and the single-scattering calculation for the period $\rho>1.3 \mathrm{~cm}$, especially for the rotation of $S_{12}$.

The mean free path of the light in a turbid medium is $1 / \mu_{T}$. As $\mu_{T}$ increases, the light, through multiple scattering, will make a greater contribution to the intensity patterns of backscattered light, which will directly influence whether a single-scattering model can be used. The histogram in Fig. 6 shows the percentage of $I_{n}$, the intensity of light detected after $n$ scatterings in the turbid medium, in the total backscattered light $I_{0}$, where the incident light is unpolarized. If the incident light is polarized with various polarization states, the histograms will be similar. The percentage of intensity from the singly scattered light is $14 \%$, and the percentage of intensity from all the multiply scattered light is $85 \%$, which means that the multiply scattered light represents a greater contribution to the intensity patterns than the singly scattered light.

Through the Monte Carlo simulation with the above parameters, the remaining degree of polarization of backscattered light comes mainly from light that has been scattered fewer than 10 times in the medium, which agrees with the results of Ambirajan and Look. ${ }^{6}$ The patterns of the Mueller-matrix elements that characterize the polarization property of the sample also come mainly from those weakly 




Fig. 9. Monte Carlo simulated rotation angle of backward Mueller-matrix element $S_{21}$ for high scattering in a turbid medium containing glucose; $\mu_{s}$ is $100 \mathrm{~cm}^{-1}, \mu_{a}$ is $10 \mathrm{~cm}^{-1}$, and the thickness of the sample $h$ is $0.04 \mathrm{~cm}$. Solid curves, linear fit.

scattered photons. The backscattering Muellermatrix patterns of singly scattered light and multiply scattered light were simulated separately. Figures 7 (a) and 7(b) show the rotation angles of patterns $S_{12}$ and $S_{21}$, respectively, where results of singly scattered light and multiply scattered light are compared with the predicted results from the single-scattering approximation model. It is interesting that for singly scattered light and multiply scattered light the rotation angles of the pattern $S_{21}$ are quite similar, especially when the source-detector distance is small, though the patterns from multiply scattered light are weaker than the patterns from singly scattered light (a weak pattern means that the contrast between the intensities at different positions of the pattern is small). For matrix element $S_{12}$, the difference in the rotations is obvious for different orders of scatters, especially when scattering coefficient $\mu_{s}$ of the turbid medium is high.

The changes in rotation angles of elements $S_{21}$ with changes in glucose concentration $\alpha$ were simulated by the Monte Carlo (symbols) method and the single-scattering (solid curves) model, respectively, as shown in Fig. 8. Here different detection positions from the incident point are considered (sourcedetector distances $\rho$ are $0.2,0.4,0.6$, and $1.0 \mathrm{~cm}$ ). The two simulation methods are in good agreement and show that the rotation degrees have a linear relationship to $\alpha$.

For highly scattering turbid media, there still exists a linear relationship between the angle of rotation of the Mueller-matrix elements and the concentration of glucose, although the singlescattering model cannot precisely predict the degree of rotation on this condition. For example, we simulated backscattering Mueller-matrix element $S_{21}$ for a turbid medium with scattering coefficient $\mu_{s}=100$ $\mathrm{cm}^{-1}$, absorption coefficient $\mu_{a}=10 \mathrm{~cm}^{-1}$, anisotropy $g=0.134$, thickness $h=0.04 \mathrm{~cm}$ and transport mean free path $l_{t}{ }^{\prime}=1 /\left[\mu_{a}+\mu_{s}(1-g)\right]=0.010 \mathrm{~cm}$. The degree of rotation as a function of the concentration of glucose is shown in Fig. 9 for various source-detector distances $(\rho=0.03,0.06,0.12 \mathrm{~cm})$, which are several times greater than $l_{t}{ }^{\prime}$. Therefore the matrix patterns considered here are from the light reflected in the diffusion regime.

To make the rotation visible in the simulation, as well as for convenience of comparison, in our discussion we used glucose concentrations much higher than physiological glucose levels. However, we expect that the proportionate relationship between the polarization rotations of patterns and the glucose concentration holds true for lower glucose levels as well because the change of backward Mueller-matrix patterns is linearly related to the rotation of the polarization plane, which further has a linear relationship to the concentration of glucose. All the equations, no matter whether they apply to the single-scattering model or the Monte Carlo simulations, show this linear relationship between the rotations of matrix elements and the concentration of glucose. If the concentration of glucose is at a typical physiological level $(80 \mathrm{mg} / \mathrm{dL})$, the sourcedetector distance is $0.12 \mathrm{~cm}$, and the other parameters are the same as those in the simulation of Fig. 9, the predicted rotation angle of matrix element $S_{21}$ will be $3.0 \times 10^{-4}$ degree. Although the detection of such a small rotation may be difficult, it is still possible if some highly sensitive methods are designed.

\section{Conclusion}

We have shown that a novel theoretical model, a single-scattering approximation based on scattering of incoherent light from spheres, can at least qualitatively emulate the patterns of a backscattering Mueller matrix in a turbid medium. With this model it is possible to analyze and predict the change in matrix patterns caused by glucose in human tissues and in the nonscattering aqueous humor of the eye. The single-scattering model is quantitatively compared with a more-accurate Monte Carlo model.

Glucose molecules, which are chiral materials, have the distinctive property of being able to rotate the polarization plane of linearly polarized light. As a result, the matrix patterns of turbid media with glucose vary according to glucose concentration. We have discussed the rotation of elements $S_{12}$ and $S_{21}$ by using both Monte Carlo and single-scattering methods. Agreement between the two methods was satisfactorily achieved in the nondiffusion regime, but the single-scattering model becomes inaccurate in the diffusion regime. We predicted, using the Monte Carlo model, that the rotation angles of the Mueller-matrix elements are proportional to the concentration of glucose in turbid media, no matter whether in the nondiffusion or the diffusion regime. This study provides insights into ways in which chiral molecules such as glucose affect the propagation of polarized light in turbid media. 
This study was sponsored in part by National Institutes of Health grants R01 CA71980 and R21 CA83760, National Science Foundation grant BES9734491, and Texas Higher Education Coordinating Board grant 000512-0123-1999.

\section{References}

1. S. L. Jacques, L. H. Wang, D. V. Stephens, and M. Ostermeyer, "Polarized light transmission through skin using video reflectometry: toward optical tomography of superficial tissue layers," in Lasers in Surgery: Advanced Characterization, Therapeutics, and Systems VI, R. R. Anderson, ed., Proc. SPIE 2671, 199-220 (1996).

2. A. H. Hielscher, J. R. Mourant, and I. J. Bigio, "Influence of particle size and concentration on the diffuse backscattering of polarized light from tissue phantoms and biological cell suspensions," Appl. Opt. 36, 125-135 (1997).

3. M. J. Rakovic, G. W. Kattawar, M. Mehrubeoglu, B. D. Cameron, L. V. Wang, S. Rastegar, and G. L. Cote, "Light backscattering polarization patterns from turbid media: theory and experiment," Appl. Opt. 38, 3399-3408 (1999).

4. G. Yao and L.-H. Wang, "Two-dimensional depth-resolved Mueller matrix characterization of biological tissue by optical coherence tomography," Opt. Lett. 24, 537-539 (1999).

5. S. L. Jiao, G. Yao, and L.-H. Wang, "Depth-resolved twodimensional Stokes vectors of backscattered light and Mueller matrices of biological tissue by optical coherence tomography," Appl. Opt. 39, 6318-6324 (2000).

6. A. Ambirajan and D. C. Look, "A backward Monte Carlo study of the multiple scattering of a polarized laser beam," J. Quantum Spectrosc. Radiat. Transfer 58, 171-192 (1997).

7. A. H. Hielscher, A. A. Elick, J. R. Mourant, and I. J. Bigio, "Biomedical diagnostic with diffusely backscattered linearly and circularly polarized light," in Biomedical Sensing, Imaging, and Tracking Technologies II, R. A. Lieberman, T. VoDinh, and G. G. Vurek, eds., Proc. SPIE 2976, 298-305 (1997).

8. A. H. Hielscher, A. A. Eick, J. R. Mourant, D. Shen, J. P. Freyer, and I. J. Bigio, "Diffuse backscattering Mueller matrices of highly scattering media," Opt. Express 1, 441-454 (1997), http://www.opticsexpress.org.

9. S. Bartel and A. H. Hielscher, "Monte Carlo simulations of the diffuse backscattering Mueller matrix for highly scattering media," Appl. Opt. 39, 1580-1588 (2000).

10. G. Yao and L.-H. Wang, "Propagation of polarized light in turbid media: simulated animation sequences," Opt. Express 7, 198-203 (2000), http://www.opticsexpress.org.

11. B. D. Cameron, M. J. Rakovic, M. Mehrubeoglu, G. Kattawar, S. Rastegar, L. V. Wang, and G. L. Cote, "Measurement and calculation of the two-dimensional backscattering Mueller matrix of a turbid medium," Opt. Lett. 23, 485-487 (1998).

12. M. J. Rakovic and G. W. Kattawar, "Theoretical analysis of polarization patterns from incoherent backscattering of light," Appl. Opt. 37, 3333-3338 (1998).

13. M. Kohl, M. Cope, M. Essenpreis, and D. Bocker, "Influence of glucose concentration on light scattering in tissue-simulation phantoms," Opt. Lett. 19, 2170-2173 (1994).

14. J. S. Maier, S. A. Walker, S. Fantini, M. A. Franceschini, and E. Gratton, "Possible correlation between blood glucose concentration and the reduced scattering coefficient of tissues in the near infrared," Opt. Lett. 19, 2062-2064 (1994).

15. J. T. Bruulsema, J. E. Hayward, T. J. Farrell, M. S. Patterson, L. Heinemann, M. Berger, T. Koschinsky, J. SandahlChristiansen, H. Orskov, M. Essenpreis, G. SchmelzeisenRedeker, and D. Bocker, "Correlation between blood glucose concentration in diabetics and noninvasively measured tissue optical scattering coefficient," Opt. Lett. 22, 190-192 (1997).

16. M. Mehrubeoglu, N. Kehtarnavaz, S. Rastegar, and L. V. Wang, "Effect of molecular concentrations in tissue-simulating phantoms on images obtained using diffuse reflectance polarimetry," Opt. Express 3, 286-297 (1998), http://www. opticsexpress.org.

17. G. X. Zhou and J. M. Schmitt, "Sensitive detection of optical rotation in liquids by reflection polarimetry," Rev. Sci. Instrum. 64, 2801-2807 (1993).

18. G. L. Cote, M. D. Fox, and R. B. Northrop, "Noninvasive optical polarimetric glucose sensing using a true phase measurement technique," IEEE Trans. Biomed. Eng. 39, 752-756 (1992).

19. B. D. Cameron and G. L. Cote, "Noninvasive glucose sensing utilizing a digital closed-loop polarimetric approach," IEEE Trans. Biomed. Eng. 44, 1221-1227 (1997).

20. C. Chou, Y. C. Huang, C. M. Feng, and M. Chang, "Amplitude sensitive optical heterodyne and phase lock-in technique on small optical rotation angle detection of chiral liquid," Jpn. J. Appl. Phys. 36(1), 356-359 (1997).

21. C. Chou, C. Y. Han, W. C. Kuo, Y. C. Huang, C. M. Feng, and J. C. Shyu, "Noninvasive glucose monitoring in vivo with an optical heterodyne polarimeter," Appl. Opt. 37, 3553-3557 (1998).

22. C. Pu, Z. H. Zhu, and Y. H. Lo, "A surface-micromachined optical self-homodyne polarimetric sensor for noninvasive glucose monitoring," IEEE Photon. Technol. Lett. 12, 190-192 (2000).

23. B. P. Ablitt, K. I. Hopcraft, K. D. Turpin, P. C. Y. Chang, J. G. Walker, and E. Jakeman, "Imaging and multiple scattering through media containing optically active particles," Waves Random Media 9, 561-572 (1999).

24. R. C. N. Studinski and I. A. Vitkin, "Methodology for examining polarized light interactions with tissues and tissuelike media in the exact backscattering direction," J. Biomed. Opt. 5, 330-337 (2000).

25. L. D. Barron, Molecular Light Scattering and Optical Activity (Cambridge U. Press, London, 1982).

26. H. C. van de Hulst, Light Scattering by Small Particles (Dover, New York, 1981).

27. L. H. Wang, S. L. Jacques, and L. Zheng, "MCML-Monte Carlo modeling of light transport in multilayered tissues," Comput. Methods Programs Biomed. 47, 131-146 (1995).

28. D. R. Lide, ed., CRC Handbook of Chemistry and Physics, 79th ed. (CRC Press, Boca Raton, Fla., 1998), pp. 3-12 and 8-64. 